\title{
Analysis techniques and models for resource optimization in Wireless Sensor/Actuator Network environment
}

\author{
Salvatore F. Pileggi, Carlos E. Palau, Manuel Esteve \\ ETSIT. Universidad Politécnica de Valencia \\ Camino de Vera S/N, 46022 Valencia, Spain \\ salpi@doctor.upv.es, \{cpalau, mesteve\}@dcom.upv.es
}

\begin{abstract}
In the last few years, WSN has been object of an intense research activity that has determined an important improvement by technologic and computation point of view both. The notable level got and the increasing request of applications designed over Sensor Networks make WSN commercial diffusion next to be a fact. Limited resource orientation and high level application requirements result in a number of key open issues, such as Resource Optimization and Quality of Service. These last two issues require an important preliminary phase of analysis and evaluation that can provide the designer with knowledge of important relationships between parameters design and application desired characteristics. Mathematical models of local resource (node), of network influence on single resource, of QoS requests, and related analysis techniques to determine not only "how much" but also "in which way" resources are expensed are proposed in this paper.
\end{abstract}

Keywords: Resource Analysis, Resource Evaluation and Optimization, Wireless Sensor Network, Quality of Service.

\section{Introduction}

The intense research activity of the last few years has determined an important improvement for WSN [1] by technologic and computation point of view. The last generation of sensor/actuator nodes, even if limited resource oriented in terms of power, is provided with ad-hoc miniaturization of advanced micro-processors, high level memory of important size, high level and low power communication possibilities based on several standards, very sophisticated sensor boards and high level operating systems. The technologic level of basic nodes reflects an important global improvement for systems designed on WSN that determines great research and commercial interest. Application level and commercial requirements address a number of key issues (and related challenges) that can globally referred as Quality of Service (QoS) requirements [2]. Some of these issues (security, reliability) both with some aspects related to compatibility could represent the real key issues for commercial diffusion of WSN in the next future. The dominant theme that characterizes the deployment of application on WSN is the optimal middleware between increasing application requests and (limited) resource optimization. Architectures, related topologies and associated mechanisms are always more 
complex and, in the great part of cases, imply a number of tradeoffs that could be in contrast between them (scalable/reliable routing or high performance routing? e.g.).As consequence, classic analysis techniques for evaluation and optimization of resource, even if not obsolete, can be considered, if related to high complexity of networks, as not suitable and, some times, not precise. These aspects advise to reconsider the term "resource optimization", generally referred, in WSN environment, "simply" as energy consumption minimization. This last concept remains generally valid but it has to be particularized into a great set of environments, requirements and characteristics completely different between them (hard real time and non real time, for example). Classic approaches are oriented to answer the question "how much?". This should be considered only as the first step and integrated with other analysis steps that should be the answer for two other questions: "in which way?" and "is system optimized in according with a number of desired characteristics?". The first issue is mainly related with network design in function of QoS desired characteristics; the second point should have a comparative approach oriented to resource optimization. This work would provide a basic and extensible analysis framework for high computing WSN. The first part of the paper is focused on resource model; theorist and general assumptions are related to concrete motes [3] and to external influence in function of QoS structures and mechanisms. Some aspects, related to analysis and optimization of resource, are described and, finally, some simulation results related with three different scenarios are showed.

\section{Related Work}

WSN is a WPAN composed by particular computational nodes provided with sensing/actuating capacity and characterized by limited resource. Its peculiar characteristics naturally orient the research on WSN to resource management/optimization. There is a considerable number of works oriented to modify and adapt mechanisms and schemes designed for traditional Wireless Networks to limited resource environment; at the same time, there are several works focused on guarantee one or more QoS properties [6] in according with resource optimization. If level considered is referred to the last generation of WSN, probably there is not an unique model that resumes, in complete and global way, the problem of resource optimization in function of a number of $\mathrm{QoS}$ desired characteristics and related tradeoffs and challenges. This work would be an example of global analysis oriented to provide the designer with an important tool for evaluation and comparison between them of different and complex architectures and solutions.

\section{Resource Model}

The analysis proposed is first focused on single computational node, considered as active part of a number of network tasks; than, node is contextualized and external influence is related with local resource expense. Moreover, information related to single nodes provides an advanced and abstract mathematical point of view of 
considered platform. Analysis is supposed to be event discrete and so mathematical expressions and relationships are always related to generic event $i$.

\subsection{Node Resource}

Node model considered and referred to as Node Resource is provided with five different resources:

- Computational Resource

- Storage Resource

- Communication Resource

- Sensing Resource

- Actuating Resource

As reference, the node is supposed to be provided with the same technologic level of MICA2 family [3]. Every resource of a node really contributes in different way to power consumption. The power consumed from event zero until a generic event $i$ is defined in (1); $P_{i}$ is power consumption related with single event $\mathrm{i} ; P_{\max }$ is maximum battery capacity; $\mathrm{k}$ is simply a scale factor.

$$
\text { Power }_{k}=k / P_{\max } * \sum_{i=0}^{k} P_{i}
$$

A node is supposed as provided of a sleep/operate protocol that works in according with the activity diagram showed in Figure 1. The "natural" state for a sensor node is "Sleep"; in this state the sensor is in very low cost mode and its power expense can be supposed next to be zero; its only activity is waiting for an external or internal event; if an event, internal or external, is generated, the sensor state becomes "Operate". This is an active state and power expense must be considered as important. When all activities related with the interest event finish, sensor state becomes "Sleep".

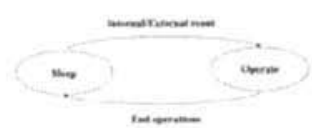

Figure 1: Sleep/Operate protocol activity.

Figure 11: HDC analysis; TopC density diagram.

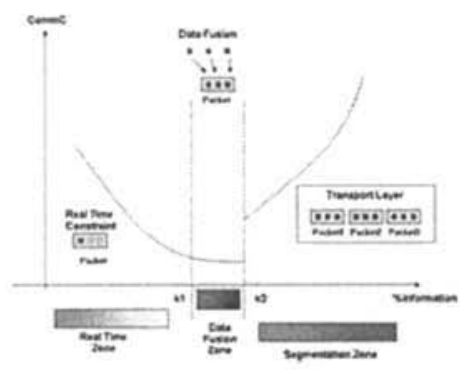

Figure 2: Transmission zones.

Active/Sleep Factor, Y, is defined in (2) and expresses the cost of Sleep/Operate protocol; $\mathrm{Y}>1$ is the cost of transition Sleep $\rightarrow$ Operate. The cost of contrary transition (Operate-> Sleep) is considered to be as no influent for analysis objectives.

$$
Y=\left\{\begin{array}{l}
\text { status }==" \text { operate } \Rightarrow Y=0 \quad \text { (2) } \quad \text { Cost }_{\text {micro-vent }}=f(\text { parameters })=\text { equation } \\
\text { status }==" \text { sleep" } \Rightarrow Y>1
\end{array}\right.
$$




$$
\text { Cost }_{\text {computation }}=f(n-\text { operations })=Y+k_{1} * n
$$

A specific cost function is defined for all micro events (internal an external), in accordance with the general model of (3). Computational cost is supposed to be proportional with the number of macro operations, as defined in (4) $\left(k_{1}\right.$, scale factor). Receive cost is supposed as proportional with the percentage of information of received message (5). Transmission cost, defined in (6), is supposed proportional with percentage of information too.

$$
\begin{gathered}
\text { Cost }_{\text {receive }}=f(\% \text { Info })=Y+k_{2} * \% \text { Info } \\
\% \text { Info }_{\text {SizeOf }(\text { Info }) /(\text { SizeOf }(\text { Info })+\text { SizeO } f(\text { Head }))} \\
\text { Cost }_{\text {transmission }}=f(\% \text { Info })=k_{3} * \% \text { Info }
\end{gathered}
$$

When a message is received, sensor node must verify if receiver is itself (preprocess) or not. Pre-process event happens always after a receive event and, so, when sensor state is "operate" $(\mathrm{Y}=0)$. Pre-process cost is assumed to be constant (7).

$$
\text { Cost }_{\text {pre-process }}=\text { const (7) } \quad \text { Cost }_{\text {capture }}=Y+\text { const (8) } \text { Cost }_{\text {actuate }}=\text { const }
$$

Also capture cost (8) is assumed as constant but it can happen when sensor node is in sleep or operate mode both. Actuating event is normally a consequence of a data process ad so it can happen only when sensor node status is "operate" $(\mathrm{Y}=0)$. Its cost is defined in (9). A node of MICA2 family [3] is considered as reference. More concretely, MICAz [3] platform is considered; MICAz has the same characteristics of MICA2 but it uses ZigBee technology [4] (based on IEEE 802.14.5 standard) that guarantee great efficiency in low power communication.

\subsection{Network Influence on Node Resource}

Network model considered is service oriented: the WSN is a unique virtual resource that globally provides a service; service provided is really the result of a number of tasks; every task can be represented by an activity of a unique sensor or by the activities of a group of cooperating nodes. However, and in whichever topology, global service is the result of a co-operation between all computational nodes (eventually with different "roles"), one or more base stations, and, eventually, gateway components. The section goal is to define a mathematical relationship between energy consumption of a node and external influence. This information also defines the "role" of the node into the system. External influence could really depend by a considerable number of factors, role and geographic/logic location of the node first of all. The main idea is to provide the designer of an analysis technique, based on a well-defined model that should provide a deepened knowledge of resource expense in function of a number of network parameters. Interest parameters (at network level) are considered:

- Communication efficiency: it depends mainly by application characteristics (hard/soft real-time, no real-time) and by throughput optimization. 
- Topology: a node is active part of a global service and, so, of one or more computational tasks. Some of its function are active (capture, for example), others passive (transit node).

- Data Process: data process implies that node status is (or becomes) "Operate" (high energy expense). If tasks are organized in co-operative way and computation level is supposed to be high, opportune strategies are advised. However, data process importance is not directly related to power expense; data process represent an important activity "map" of the network and, if required, could provide the designer with an important knowledge related, directly or indirectly, to complex tasks and planes.

- Sensing plan: every application/architecture has its own requests in terms of information capture. Sensing is, both with communication, the main function of a node in WSN. In this sense, reliability and optimization importance advises to design (if required) capture planes that could guarantee performance, fault tolerance and reliability.

- Actuating plan: if network is also provided with actuating functions, an actuating plane should be designed in accordance with other optimization planes. This issue is more relevant respect to planes that manage the events that cause the actions (capture for example).

For each parameter, a mathematic definition is proposed. A resuming five dimensions function (External Influence, EI) for these issues is defined in (10); a mono dimension version of EI (linear combination of each contributes), called $M D$ $E I$, is also defined in (11).

$$
\begin{gathered}
\quad E I_{k}=\left(\sum_{i=0}^{k} \operatorname{CommC}_{i}, \sum_{i=0}^{k} \operatorname{Top}_{i}, \sum_{i=0}^{k} \operatorname{Pr} o C_{i}, \sum_{i=0}^{k} \operatorname{CapC}_{i}, \sum_{i=0}^{k} A c t C_{i}\right) \\
M D-E I_{k}=\sum_{i=0}^{k} a^{*} b_{k i}^{*} E I_{k} \\
\left\{\begin{array}{l}
a=\text { scaleFactor } \\
b_{k i} \in[0.1] \\
k=1,2,3,4,5
\end{array}\right.
\end{gathered}
$$

As showed in Figure 2, in function of the percentage of information, three different zones can be distinguished: Real Time zone, Data Fusion/Aggregation zone, Segmentation zone. Real Time zone is characterized by information flows of little size and is so named because it is the typical environment of applications characterized by time constraints; the Fusion/Aggregation zone is the ideal work area by throughput point of view because the amount of information is considered to be optimal (high information percentage); apposite mechanisms (data fusion and aggregation) generally try to force applications to work constantly in this area; the third zone supposes a great amount of information that requires (or advises) data segmentation and related mechanisms (transport protocol, for example). Figure 2 shows proposed Communication Coefficient (CommC) too; a possible mathematical definition of CommC is proposed in (12). 


$$
E I_{k}=\left(\sum_{i=0}^{k} \operatorname{Comm}_{i}, \sum_{i=0}^{k} \operatorname{Top}_{i}, \sum_{i=0}^{k} \operatorname{Pr} o C_{i}, \sum_{i=0}^{k} \operatorname{Cap}_{i}, \sum_{i=0}^{k} A_{c t C_{i}}\right)
$$

$$
\left\{\begin{array}{l}
f_{i} \in[1, l] \\
l>1 \\
\text { init }: l=2 \\
\text { TransmissibnFailure: } l=l+1
\end{array}\right.
$$

The behavior of CommC, as showed in Figure 2, is oriented to optimize throughput and energy efficiency. However, (12) has a sufficient level of dynamism, guaranteed by a number of parameters, to describe different behaviors, for example considering a different zone as desired if system specifications advise it. When a message is received, this must be pre-processed by sensor node to establish if the message receiver is itself (active role) or not (passive role). In this second case message must be re-transmitted. Topology Coefficient (TopC), defined in (13), is an important evaluation of contribute to resource expense of passive activities for considered node. High values of TopC generally advise to increment network scalability because node activity is considered as too much passive.

$$
\begin{array}{r}
\quad \text { Top }_{i}=s c * \text { top }_{i} *\left[\text { Cost }_{\text {receive }}+\text { Cost }_{\text {pre-process }}\right] \\
\text { top }_{i}=\left\{\begin{array}{l}
\text { node.id }==\text { message.receiver } \Rightarrow \text { top }_{i}=1 \\
\text { node.id } \neq \text { message.receiver } \Rightarrow \text { top }_{i}=w, w++ \\
\text { init }: w=2 \\
s c=\text { scaleFactor }
\end{array}\right.
\end{array}
$$

Data Process Coefficient (ProC) can be defined as whichever function that has a, direct or indirect, dependence by number of elementary (or macro) operations processed or by activity time of node; an example of ProC definition is proposed in (14).

$$
\operatorname{Pr} o C_{i}=\mathrm{Dp} * \mathrm{f}(\mathrm{n})=\mathrm{Dp} * n^{2}
$$

$\mathrm{n}=$ number of macro operations

$\mathrm{Dp}=$ scale factor

Data Process expense is no relevant in the great part of cases because its contribution to energy consumption is considered as inferior to others and, moreover, it is not always easy to measure with an appropriate accuracy level. On the other hand, ProC, as data process measurement, has great importance for analysis of activities into the network. Sensing activities are expressed by Capture Coefficient $(C a p C)$ defined in (15). As showed, it is supposed that a network can be provided with independent sensing tasks (no cooperative capturing) or with cooperative sensing tasks.

$$
\operatorname{CapC}_{i}=d^{*} \operatorname{Cost}_{\text {capture }} * e^{r / s c c}
$$




$$
\left\{\begin{array}{l}
\text { IndependentSen } \sin g \text { Task } \Rightarrow r=1 \\
\text { CooperativeSen } \sin g \Rightarrow\left\{\begin{array}{l}
\text { init }: r=0 \\
\text { capture }: r++ \\
\text { endOfTask }: \text { init }
\end{array}\right.
\end{array}\right.
$$

$\mathrm{scc}=$ scale factor

$\mathrm{d}=$ scale factor

Sensing task mechanisms are considered to be expensive but, at the same time, they make architectures more oriented to peer-to-peer and can guarantee accuracy in measurements, an important improvement of network robustness, an important support for failure detection and related reconfiguration operations. Considering capture planes, an important tradeoff between QoS and resource requirements is addressed. CapC could represent an important driver factor to compare different solutions. Also actuator placement and the design of action tasks could affect entire architecture design. In this sense the radius of action (measured in hops) seems as the key factor for evaluation. Actuator Coefficient (ActC), defined in (16), is the expression of actuating influence (direct or indirect) on resource.

$$
A c t C_{i}=\operatorname{Cost}_{\text {Actuate }} * e^{h / A}
$$

$A=$ factor scale

$\mathrm{h}=$ radius of action of interested actuator

As for sensing tasks, if actuator activity is considered to be important, the design of actuating planes is advised.

\subsection{QoS influence on resource}

Quality of Service (QoS) can be so defined: "In the fields of packet-switched networks and computer networking, the traffic engineering term Quality of Service (QoS) refers to control mechanisms that can provide different priority to different users or data flows, or guarantee a certain level of performance to a data flow in accordance with requests from the application program". Quality of Service must be considered as the real key issue when, as in WSN, resource are limited or no always sufficient if related with application requests; the importance of QoS mechanisms generally increases on large scale application fields. An approach simply "best effort" (and not regulated by QoS mechanism) in WSN could mean limited deployment strategies, higher human operations (this could be not easy and generally considered to be expensive), higher number of sensors to guarantee the same service in the same conditions and lifetime and, more generally, lower "quality" (efficiency, security, reliability, and so on). QoS is normally referred to as "QoS mechanisms"; this is because there are many parameters that can determine or affect the quality of service and so more than one protocol needs to guarantee a considerable number of desired characteristics. QoS mechanisms of a WSN are largely different to mechanisms of a traditional or standard network; they are mainly (but not only) focused on the tradeoffs between QoS and Resource Efficiency. Traditional networks are affected by classic problems as dropped packets, delay, jitter, out-of-order delivery, error, that recommend to design QoS mechanisms based on "Calling" approach or "In Advance" approach or reserving resource. Some mechanisms, or better, some of approaches proposed over traditional networks, with opportune modifications, can be applied in 
WSNs too. Recently, a number of Resource Reservation mechanisms (based on softstate agents, for example) were proposed. Probably, adapting mechanism designed for standard network to WSN is not exactly the best approach because WSN has its own design parameters (specific node hardware, specific operating systems, ad-hoc protocols and, above all, topology) and its own goals that depend mainly by application characteristics (real-time, no hard real-time, hard real-time), by system scale and by others characteristic issues. All issues (design parameters, goals) are different by the correspondent issues of a standard network. In this paper, different sub issues, more concretely security, fault tolerance, robustness, reliability and throughput are globally referred to as QoS; these issues, as well as theoretically independent, can have a direct relationship or tradeoff between them, or, as general issues, an important tradeoff with energy efficiency; resuming, all QoS parameters are indirectly related by the common and central tradeoff with energy efficiency. Function $\mathrm{Y}$ is an evaluation of QoS mechanisms incidence on local resource.

$$
Y_{i}=\mathrm{f}(\mathrm{QoS})
$$

(17) should be particularized and it should explicitly represent the relationships between QoS mechanisms and node resource, modeling explicitly requirements and related tradeoffs. However, this last point, even if considered as one of central issues in resource analysis, is out of scopes of this work that is mainly focused on methodologies more than models. QoS mechanisms influence on resource is considered as a measurement of percentage of incidence on total expense.

\section{Resource Analysis}

Resource analysis (Figure 3) is articulated in three basic steps: modeling, measurement and evaluation/comparing/ optimization. The 3D function defined in (18) and its mono-dimensions version (19) could represent an important analysis instrument for WSN resource. Considering a 3D plane, $x$-axis is Power (1) and represents the real power expensed by node, $y$-axis is $Y(17)$ and represents the QoS mechanisms influence on energy expense, and, finally, z-axis is MD-EI (11) and represents external influence and the role of node in the network.

$$
\left(\text { Power }_{i}, Y_{i}, M D-E I_{i}\right)=M D-\text { eval }_{i} \quad \text { (18) } \quad \sum_{i=0}^{k}\left(\text { Power }_{i}+Y_{i}+M D-E I_{i}\right)=\text { eval }_{i}
$$

The first step is mainly related to the modeling of considered node platform on the mathematical model proposed; this implies the numeric estimation of the cost of some events/actions and the set of related scale factors; some of these estimations could result not easy or, however, not too much precise; in this last case, relationships between various events, network properties and, eventually, a number of theoretical/practices assumptions can help the designer to obtain a behavior model really next to real platform. In modeling step, optimization goal should be defined and particularized as a number of well-defined constrains or conditions over MD-EI or over some of its parameters. Modeling step is something more than a simple preliminary phase and, in some cases, could result as a hard and central step, in 
particular way in presence of complex relationships/tradeoffs; its affectivity generally conditions final results. Mechanisms, protocols and architectures analysis have, as general objective, to minimize the driver coefficient MD-EI (z-axis), as expression of the key idea of find an optimal middleware between desired characteristics and energy efficiency. Valid analysis perspectives are considered the $\mathrm{x}-\mathrm{y}$ plane (direct relationship between total power expensed and power expensed by QoS mechanisms), $\mathrm{x}-\mathrm{z}$ plane (direct relationship between power expensed and network influence) and $\mathrm{y}-\mathrm{z}$ plane (direct relationship between QoS mechanisms and related activity at network level). Integral perspective $(20,21)$ could result interesting too.

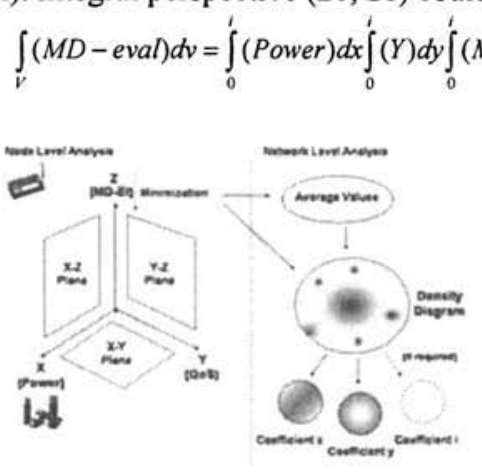

Figure 3: Resource Analysis.

$$
\int_{1}^{(e v a l) d i}
$$

Even if considering external influence, node role in the network, passive/active tasks of node in the network, the analysis limited to a single node provides a local perspective that doesn't permit an efficient evaluation/optimization step. Evaluation and optimization of resource are referred at network (or sub-network) level. Network analysis results more effective and indicative if single node measurements are extended at local area, considering values related to different nodes and mathematical/statistic elaborations of information, such as average values in certain conditions; the final result of measurement step is the definition of density diagrams of resuming evaluation functions, or, better, the definition of density diagrams related to each interest coefficient of MD-EI. Independence of optimization goal, analysis can be referred to all sensors, providing an Area Density Diagram (ADD) or can be restricted only at a number of key nodes, providing a Logic Density Diagram (LDD). ADD can provide an optimal perspective of the system relatively to aspect directly or indirectly related to traffic, communication, large scale, routing; LDD results more direct if related to the evaluation of tasks, gateway service and hierarchical organization. As general assumption, the global point of view provided by both these diagrams could result as important in the phase of evaluation/optimization that should result as simplified. The minimization of MD-eval (resource optimization) can or cannot have a direct relationship with Power; the proportional decreasing of Power represent probably the desired situation; however, in a great number of situations, resource optimization can result in a better organization and management of resource that has a little (or not appreciable) effect over Power but a considerable impact on the improvement of system time-life. 


\section{Simulation}

Three different scenarios will be analyzed. These architectures should be considered as examples but, at the same time, they represent some of most common and relevant topologies in WSN deployment context. Figure 4,a (Basic Cell, BC) represents a multi-sink architecture composed by a number of basic cells formed by a sink and several sensor nodes. Communication into the cell is mono-hop and nodes simply provide independent sensor tasks and so its role is always considered as active. Every zone has its own keep-live system to detect eventual failure situations. Sinks are supposed as to be organized in hierarchical way and provided with both high and low power (and range) communication capacity.

\begin{tabular}{lcccc}
\hline \multicolumn{1}{c}{ Configuration } & Communication & Sensing & Actuator & QoS \\
\hline BC & Mono-Hop & Independent & No & $1 \%$ \\
HDC & Multi-Hop & Independent & $1-5 \mathrm{Hops}$ & $5 \%$ \\
CWSN & Multi-Hop & Independent & $1-2 \mathrm{Hops}$ & $6 \%$ \\
& & Cooperative & & \\
\hline
\end{tabular}

Table 1. Simulation Configuration.

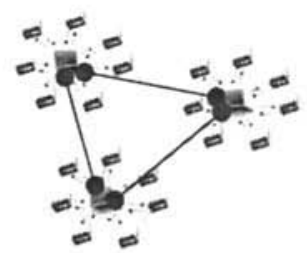

(a)

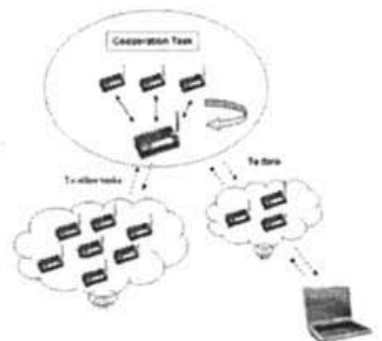

(b)

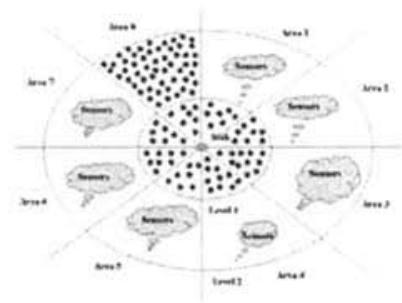

(c)

Figure 4: BC (a), HDC (b), CWSN (c).

The second scenario, showed in Figure 4(b), is the High Density Cell (HDC). HDC is characterized by a unique sink and by a great number of sensor nodes uniformed distributed into considered area. Communication is multi-hop and each sensor node has really two important roles: it is part of communication network as transit node (passive role) and it has an independent sensor task (active role). Considering topology, a more advanced Keep-live system, and consequently characterized by a higher resource request, is provided too. The last scenario (Figure 4,c) shows Cooperative WSN (CWSN) that proposes a mono-sink architecture with the same characteristics of HDC but provided with Sensor Task Structures [5]; these mechanisms provides the system with co-operative sensing that guarantees great accuracy in measurements and an important support for fault detection and related reconfiguration. In CWSN architecture, only a limited number of sensor nodes are part of cooperative sensor tasks; the others provide an independent sensing service. Results are showed in Figure 6 (Power), Figure 7 (contextualized analysis) and Figure 9 (MD-eval). If analysis is limited to classic approach (energy consumption evaluation that is represented by Power in proposed model), HDC and CWSN result 
as no distinguished and, moreover, the knowledge related to network activities results as very limited.

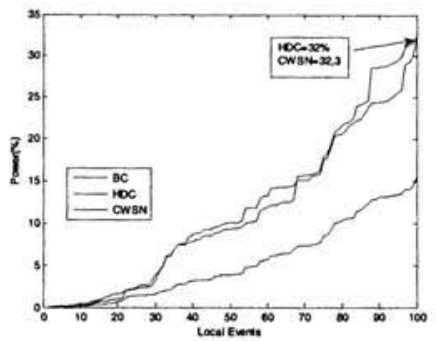

Figure 6: Power.

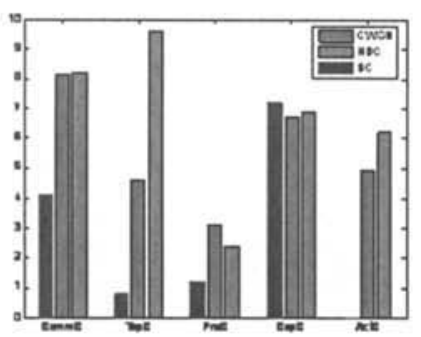

Figure 7: Network Influence/Node role

An extended analysis, that considers power consumption both with a model for the evaluation of network influence (Figure 7) on local resource and, consequently, the determination of the node "role" in the network, could represent a key issue to resource evaluation and optimization of relationship between performance and resource request. Measurements related to $\mathrm{BC}$ architecture analysis detect limited power expense (Figure 6) and optimized communication (Figure 7). These issues are the main motivations that advise hierarchical organizations (Figure 4,a). If this architecture is compared with another that guarantees the same service in the same area, gateway nodes (base stations in this case) properties must be considered too.

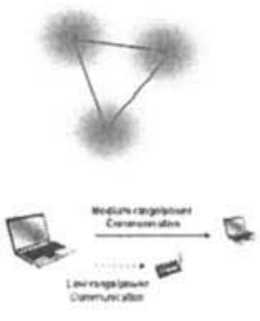

Figure 10: $\mathrm{BC}$ analysis. CommC density diagram.

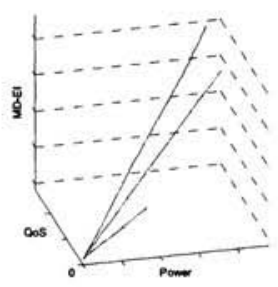

Figure 9: MD-eval.

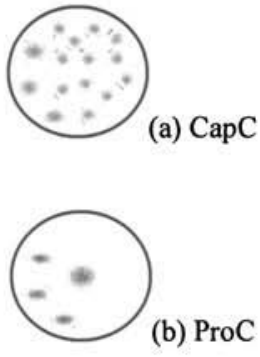

Figure 8: CWSN analysis.

The best and simple way to provide information in this sense is to consider CommC density diagram (Figure 10). In the few cases in which base station is oriented to limited resource too, this configuration could result as inappropriate. The diagram shows the real "architecture" cost: sensor gateway nodes have the real ownership of communication. This last point must be considered at the time of a comparison. Figure 7 shows a great passive role for sensor nodes of HDC architecture. Geographic nodes distribution over considered area is uniform (Figure $4, c)$ and so nodes next to sink are really very loaded (Figure 5). TopC density diagram (Figure 11) shows this role considered as too much passive. Moreover, energy consumption is directly related with communication and so the power of nodes next to 
sink rapidly decreases and could rapidly isolate certain parts of network with very negative impact over network lifetime. This advises a topology with no uniform geographic distribution: nodes density should be inversely proportional with sink distance and routing protocol strategy should be oriented to scalability. A combined analysis of CapC (Figure 8,a) and ProC (Figure 8,b) density diagrams related to CWSN permits to have a map of activities (sensing in this case). If analysis is limited to sensing activities (CapC), cooperative sensor tasks are no localizable or localizable with difficulty. As showed, integrating the analysis with information related to data process (activity of sensor), the localization results very easy.

\section{Conclusions and Future Work}

An approach for analysis of modern WSNs resource in function of architecture, desired characteristics and related constraints was proposed. Theoretical assumptions and models were related with characteristics of concrete sensor nodes and with realistic and consistent scenarios. Analysis technique proposed results as effective considering both elementary and complex situations; moreover, it can optimally support the designer in presence of critical tradeoffs and challenges. Simulation results can be considered as in accordance with theoretic assumptions. Future extensions of model are mainly related to the explicit consideration of large scale contexts and, mainly, to the integration of proposed models with a well-defined QoS model that should explicitly represent the relationships between QoS mechanisms and requirements on both local and global resource.

\section{References}

1. I. Akyildiz, W.Su, Y.Sankarasubramaniam, E.Cayirci, A survey on Sensor Network, IEEE Communication Magazine, August 2002.

2. Dazhi Chen and Pramod K. Varshney, QoS Support in Wireless Sensor Networks: A Survey, International Conference on Wireless Sensor Network 2004, Las Vegas, Nevada, USA.

3. CrossBow Technology, www.xbow.com.

4. ZigBee Alliance, www zigbee.org.

5. M. Ilyas, and I. Mahgoub, Handbook of Sensor Networks: Compact Wireless and Wired Sensing Systems, CRC Press, 2004.

6. Yuanli Wang, Xianghui Liu, Jianping Yin, Requirements of Quality of Service in Wireless Sensor Network, International Conference on Networking, International Conference on Systems and International Conference on Mobile Communications and Learning Technologies (ICNICONSMCL'06).

7. Dazhi Chen and Pramod K. Varshney, QoS Support in Wireless Sensor Networks: A Survey, International Conference on Wireless Sensor Network 2004, Las Vegas, Nevada, USA.

8. Jan Beutel, Matthias Dyer, Lennart Meier, Matthias Ringwald, Lothar Thiele, Next-Generation Deployment Support for Sensor Networks, 2nd International Conference on Embedded Networked Sensor Systems, Baltimore, MD, USA, November 2004.

9. Vassileios Tsetsos, George Alyfantis, Tilemahos Hasiotis, Odysseas Sekkas, and Stathes Hadjiefthymiades, Commercial Wireless Sensor Networks: Technical and Business Issues, Second Annual Conference on Wireless On-demand Network Systems and Services (WONS'05).

10. S. Tilak, N. B. Abu-Ghazaleh and W. Heinzelman, Infrastructure Tradeoffs for Sensor Networks, WSNA02, September 2002, Atlanta, Georgia, USA. 